\title{
Role of IL-18 in transplant biology
}

\author{
Chen Liu ${ }^{1, a}$, Juntao Chen ${ }^{1, a}$, Baoqing Liu ${ }^{1, a}$, Shunzong Yuan ${ }^{2, \text { a }}$, Dawei Shou ${ }^{3}$, Liang Wen ${ }^{1}$, \\ Xiaoying $\mathrm{Wu}^{4}$, Weihua Gong ${ }^{1}$ \\ ${ }^{1}$ Department of General Surgery, Second Affiliated Hospital of School of Medicine, Zhejiang University, Hangzhou City, People's Republic of China \\ ${ }^{2}$ Department of Laboratory Medicine, the PLA 307 Hospital, Beijing, People's Republic of China \\ ${ }^{3}$ Intensive Care Unit, Zhejiang Hospital, People's Republic of China \\ ${ }^{4}$ Department of Thyroid and Breast Surgery, Tongde Hospital of Zhejiang Province, Hangzhou City, People's Republic of China \\ Correspondence \\ <weihuagong@zju.edu.cn>
}

Accepted for publication June 08, 2018

To cite this article: Liu C, Chen J, Liu B, Yuan S, Shou D, Wen L, Wu X, Gong W. Role of IL-18 in transplant biology. Eur: Cytokine Netw. 2018; 29(2): 48-51 doi:10.1684/ecn.2018.0410

\begin{abstract}
Since pro-inflammatory cytokine IL-18 and its receptor (IL-18R) are closely involved in regulating both adaptive and innate immune responses, it is conceivable that they might play an important role in organ transplantation. IL-18 can stimulate lymphocytes to produce the IFN- $\gamma$ and regulate macrophage activity, thereby increasing the expression of proinflammatory cytokines including IL-1及, IL-6, CCL4 (macrophage inflammatory protein-1 $\beta$ ), CXCL2 (macrophage inflammatory protein-2), and CCL2 (monocyte chemotactic protein-1). Nevertheless, the IL-18 signaling pathway and its underlying mechanisms remain obscure in transplant biology. This review is to summarize recent advances in our knowledge about the IL-18 signaling pathway and to analyze their functions in transplant-related biology. It was found that IL-18/IL-18R signaling pathway contributed to vascular transplantation, ischemmia/reperfusion, acute kidney injury, and acute rejection of kidney/liver/heart transplantation. IL-18 was a potential CYP3A expression modulator and was capable of affecting tacrolimus pharmacokinetics. Neutralizing IL-18 by its inhibitor IL-18 binding protein could efficiently suppress the production of injury-associated cytokines such as IL-6, TNF- $\alpha$, IFN- $\gamma$, CXCL10 (IFN- $\gamma$-inducible protein10), and CX, CL1 (fractalkine) and improve allograft function. Blockade of IL-18 signaling could regulate cardiomyocyte apoptosis and inhibit Th17 cells differentiation. Alteration of IL-18 levels was suggested as a biomarker for predicting ongoing allograft outcome. All these activities could deepen our understanding of immunobiological role of IL-18 and its receptor in the field of organ transplantation. Intervention of IL-18 signaling pathway might be utilized as a therapeutic strategy in clinic.
\end{abstract}

Key words: IL-18, inflammatory cytokines, organ transplantation

Interleukin-18 (IL-18) is a member of the interleukin-1 family. It is initially called IFN- $\gamma$ (interferon- $\gamma$ ) inducing factor and plays an important role in both innate and adaptive immunobiology [1]. IL-18 receptor (IL-18R) is expressed on Th1 lymphocytes and, therefore, IL-18 is capable of potently inducing Th1 responses [2]. In addition, IL-18 bearing pleiotropic effects can induce Th2 responses with no need of IL-12 [3]. Boost of Th1 and $\mathrm{Th} 2$ immune responses can elicit various cytokines including IL- $1 \beta$, IL-2, TNF- $\alpha$ (tumor necrosis factor- $\alpha$ ), IFN- $\gamma$, adhesion molecules, and apoptosis factors (figure 1) [4-6]. IL-18 can be expressed by a wide range of immune cells (T cells, B cells, NK-T cells, neutrophils), although the main source of IL-18 is from the activated macrophages [2]. Other non-immune cells can also express IL-18 such as intestinal and airway epithelial cells, keratinocytes, airway epithelium, corneal epithelial cells, renal tubular epithelial cells [7]. The main role of IL-18 is to crucially stimulate lymphocytes to produce the IFN- $\gamma$ [5]

\footnotetext{
a equal contribution
}

and regulate macrophages activity, thereby increasing the expression of pro-inflammatory cytokines including IL6 , IL-1 $\beta$, CCL4 (macrophage inflammatory protein-1 $\beta$ ), CXCL2 (macrophage inflammatory protein-2), and CCL2 (monocyte chemotactic protein-1) [8]. IL-18 in conjunction with IL-12 can stimulate the production of IFN- $\gamma$ by $\mathrm{B}$ and $\mathrm{T}$ cells and potentiate the cytolytic activity of natural killer (NK) cells, and induce Th1 and Th2 responses (figure 1) [9, 10]. In addition, IL-18/IL-1 $\beta$ signaling pathway can trigger the transcription of multiple inflammatory genes via the activation of nuclear factor $\kappa B$ and transcription activator-1. Subsequently, pro-inflammatory cytokines, chemokines, adhesion molecules, and colonystimulatory factors are induced to enhance leukocyte infiltration [11]. These aforementioned cytokines are capable of promoting acute cellular rejection in solid organ transplantation. Strikingly, damage caused by IL-18 does not depend upon CD4+T cells or neutrophils [12].

Over-production of IL-18 can potently cause a variety of severe inflammatory disorders such as autoimmune diseases, transplant rejection, and ischemia/reperfusion injury [13]. In clinic, it was observed that IL-18 was closely 


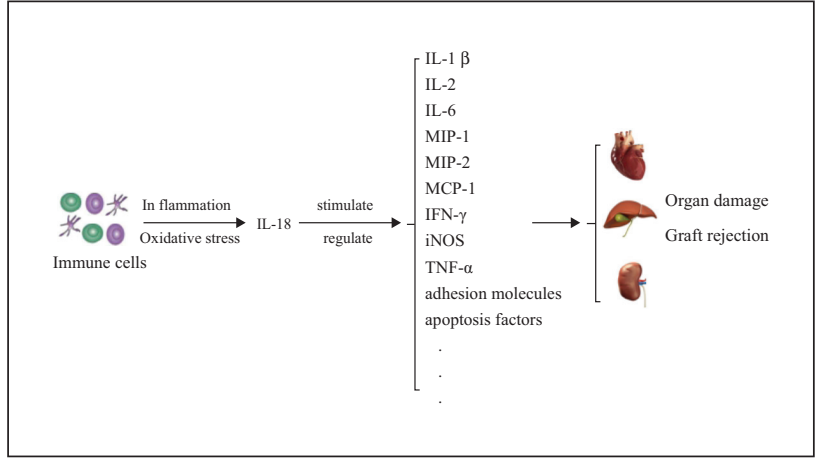

Figure 1

Scheme for the role of interleukin (IL)-18 in the elicitation of oxidative stress and proinflammation causing organ damage and graft rejection in organ transplantation.

associated with various human diseases such as inflammatory bowel disease, systemic lupus erythematosus, sub-acute sclerosingpanencephalitis, rheumatoid arthritis, and allograft dysfunction posttransplantation [13]. Inhibitors of IL-18 (rIL-18BP (binding protein), GSK 1070806, ABT-325) were successfully developed for clinical studies especially for chronic inflammation scenarios [14]. This present review attempts to analyze the roles of IL-18 signaling pathway in transplant biology, which may shed light on unveiling allogeneic immunoresponses and transplant tolerance induction.

\section{ROLE OF IL-18 IN VASCULAR TRANSPLANTATION}

Both IL-18 and IL-1 $\beta$ belong to the same family of IL1. They have a homologous amino acid sequence and share specific biological functions [15]. As important proinflammatory cytokines, both of them are closely involved in the pathophysiology of vein graft remodeling [11]. As a vein was engrafted into an artery, vascular smooth muscle cells (VSMCs) turned massively necrotic and monocytes infiltrated into venous wall of graft. Afterwards, IL-18 and IL-1 $\beta$ were highly expressed by monocytes at 1 week after vein transplantation [11]. It was found that peripheral level of IL-18 was closely associated with carotid intima-media thickness in clinic [16]. Experimental study exhibited that neutralizing IL-18 and IL-1 $\beta$ by using antagonist recombinant IL-1ra-Fc-IL-18bp could effectively suppress the activation of VSMC proliferation, cell growth-related signaling molecules, and vein graft thickening in vivo [11].

\section{ROLE OF IL-18 IN ACUTE KIDNEY INJURY (AKI)}

Acute kidney injury (AKI) is a relatively common and serious complication after liver transplantation or ischemia/reperfusion in clinic $[17,18]$. Serum IL-18 as a proinflammatory cytokine is closely associated with inflammatory events and multi-organ dysfunction [19]. Liver ischemia and reperfusion (1 hour and 4 hours respectively) could induce expression of IL-18, IL-6, IL-1 $\beta$, and TNF- $\alpha$, leading to renal damage [18]. It was observed that serum IL-18 and urine IL-18, IL-6, CXCL8 (IL-8), and NGAL levels in patients were significantly increased in AKI within the first 24 hours after liver transplantation [17]. The alteration of urine and the serum level of interleukin-18 were involved in ischemia-reperfusion injury and acute kidney ischemia, suggesting that they could be utilized as an early predictor for acute kidney injury (AKI) after liver transplantation [19]. Indeed, pretreatment with IL-18BP could attenuate renal damage with antioxidant and anti-inflammatory effects $[17,18]$. Recently, clinical data displayed that combined use of IL18 and endothelin- 1 would better predict AKI at the early stage after liver transplantation [20].

\section{ROLE OF IL-18 IN KIDNEY TRANSPLANTATION}

Experimental studies exhibited that IL-18 had a key role in rat and mouse kidney transplantation. In a mouse MHCmismatched model of acute renal rejection, IL-18 and its receptor expressions were dramatically upregulated within kidney allografts, whereas IL-18 and its downstream pro-inflammatory cytokines (iNOS, TNF- $\alpha$, IFN- $\gamma$ ) expressions were significantly downregulated in IL-18 ${ }^{-/}$ recipients (figure 1) [21]. In a rat model of acute kidney allograft rejection, a 3-fold upregulation of IL-18 mRNA expression was detected within allografts, which were mainly from $\mathrm{ED}^{+}$macrophages [22]. Similar clinical observation was achieved, in which a significant elevation of serum IL-18 (approximately 3-fold) was found in patients with kidney rejection [23]. Further investigation revealed that epithelium of distal tubules, proximal tubules, and infiltrating leukocytes in the kidney expressed IL-18 mRNA and released mature IL-18 particularly in response to IFN- $\gamma$ and TNF- $\alpha$ [23]. In clinic, urinary IL-18 levels were apparently augmented in patients with acute tubular necrosis (ATN) and delayed graft function, therefore, which was suggested as a biomarker for proximal tubular injury in ATN [24]. A significantly increased concentration of IL-18 in urine was observed, which was considered as a biomarker for acute renal failure [5]. IL-18 in combination with NGAL (neutrophil gelatinase-associated lipocalin) was suggested as a biomarker for delayed graft function after kidney transplantation [25]. Subsequently, other researchers further confirmed these findings in clinic. Multivariate analysis exhibited that augmentation of IL-18 or NGAL might predict a need for ongoing dialysis within first week of kidney transplantation after adjusting for recipient and cold ischemia time, donor age, urine output, and serum creatinine. In addition, IL-18 and NGAL quantiles could accurately predict graft recovery up to three months later [25].

Past studies revealed that IL-18 (rs187238) gene polymorphism was not associated with kidney graft outcome after transplantation [5]. The -137C/G (rs187238) and 607C/A (rs1946518) variant alleles in the IL18 gene were not associated with creatinine clearance, implying that IL18 polymorphisms could not affect kidney function [26]. Nevertheless, it was found that an increase of frequency of the IL-18 major haplotype $-607 \mathrm{C} /-137 \mathrm{G}$ was observed in renal transplant patients [26]. Presence of AA genotype of IL-18 (rs1946518) was indicative of 2.35 higher risk of chronic rejection occurrence [5]. 
In addition, both IL-18 and IL-1 $\beta$ were potential CYP3A expression modulators. In kidney transplantation, CYP3Adependent drug disposition might be influenced by IL-18 variability in different recipients [4]. Furthermore, IL-18 was involved in the induction of various inflammatory cytokines, which also could downregulate cytochrome P450 enzyme activities and influence CYP450-dependent drug disposition. Tacrolimus as an immunosuppressant is commonly used in kidney transplantation. It was found that its concentration/dose (C/D) ratio was evidently associated with IL-18 rs1946518 gene polymorphism in the first month after kidney transplantation. IL-18 promoter polymorphisms might facilitate to individualize tacrolimus treatment based upon CYP3A5 genotype [4].

\section{ROLE OF IL-18 IN LIVER ISCHEMIA-REPERFUSION INJURY}

Ischemia-reperfusion is an inevitable process for organ transplantation, in which blood supply is restored and subsequently free oxygen radicals and proinflammatory cytokines are induced to result into graft injury. IL-18 is one of the insulting cytokines mainly responsible for producing IL-1 $\beta$ and TNF- $\alpha$ from mononuclear cells. In hepatic ischemia-reperfusion injury model, Kupffer cells were main source of IL-18 at early stage. Neutralizing IL-18 by its inhibitor IL-18 binding protein (BP) could efficiently alleviate hepatic function and inhibit generation of injury-associated cytokines such as IL- 6 , TNF- $\alpha$, and IL18. Administration of IL-18BP mitigated hepatic oxidative stress, which was well-recognized to trigger production of pro-inflammatory cytokines and cell adhesion molecules. Afterwards, hepatic pathological structure was protected [8].

\section{ROLE OF IL-18 IN LIVER TRANSPLANTATION}

In a rat liver transplant model, overexpression of IL-18BP by using adenovirus gene transfer (Adex-IL18bp) could significantly decrease serum alanine aminotransferase levels and prevent histologic hepatic injury in transplant recipients. The underlying mechanisms were that IL18BP specifically blocked the binding of mature IL-18 to its receptor and thereby suppressed IL-18-induced IFN$\gamma$ production. Pre-treatment with Adex-IL18bp caused a significant prolongation of rat liver allograft survival with a lower expression level of IFN- $\gamma$, CXCL10 (IFN$\gamma$-inducible protein10), and $\mathrm{CX}_{3} \mathrm{CL1}$ (fractalkine) [7]. However, no significant association between IL-18 ($656 \mathrm{G} / \mathrm{T}, \mathrm{rs} 1946519)$ and acute rejection of liver transplant patients was observed in clinic. As liver transplant patients were subcategorized, it was found that IL-18 TG genotype had a remarkable association with rejection in female patients compared with males. Therefore, the genotype of IL-18 TG was a sex-dependent risk factor for acute rejection episode of liver transplantation [13]. Furthermore, it was observed that IL-18 (rs1946519) expression was increased for insulin resistance posttransplantation, which might be novel important biomarker for diabetes mellitus after liver transplantation [27].

In addition, it was found that IL-18 was associated with tacrolimus pharmacokinetics. One Chinese research group utilized HRM analysis (high-resolution melting curve analysis) to study two single-nucleotide polymorphisms G-137C (rs187238) and A-607C (rs1946518) in the promoter region of IL-18 gene for tacrolimus pharmacokinetics and hepatic allograft dysfunction among human 150 liver transplant recipients. These two single nucleotide polymorphisms (A-607C and G-137C) were repeatedly associated with IL-18 promoter transcription activity. The findings indicated that IL-18 decreased tacrolimus concentration/dose (C/D) ratio post-transplantation. Higher IL-18 and lower tacrolimus concentration/dose (C/D) ratio were indicative of the risk of subsequent hepatic allograft dysfunction [6].

\section{ROLE OF IL-18 IN HEART TRANSPLANTATION}

In a mouse heart transplant model, lower inflammatory cytokines such as IL-1 $\beta$, IL-17, IL-23, and IL-18 were detected in the IL-18BP-treated mice. Th17 differentiation was suppressed in vitro and in vivo. Adoptive transfer of T cells from IL-18 binding protein treated mice could ameliorate cardiac ischemia/reperfusion myocardial injury and cardiomyocyte necrosis. Infiltration of CD4+T cells, neutrophils, and macrophages was then prevented on $24 \mathrm{~h}$ after reperfusion. In addition, blockade of IL-18 signaling could regulate cardiomyocyte apoptosis. These findings manifested a destructive role of inflammatory cytokine IL-18 in cardiac IR injury and heart transplantation [2].

\section{CONCLUSIVE REMARKS}

Neutralizing IL-18 by its inhibitor IL-18 binding protein could suppress the production of injury-associated cytokines and Th17 cells differentiation and improve allograft function. Alteration of IL-18 level might be indicative of ongoing acute kidney injury, diabetes mellitus, and allograft outcome. All these aforementioned data could deepen our understanding of immunobiological role of IL-18 and its receptor in the field of transplantation. Intervention of IL-18 signaling pathway might be utilized as a therapeutic strategy in clinic.

Disclosure. Financial support: The project was supported by the National Science Foundation for Outstanding Young Scholars of China (No. 81522006), the Fundamental Research Funds for the Central Universities (2015XZZX004-21), the National Natural Science Foundation of China (No. 81470527), Zhejiang Provincial 151 Talent Project, and Zhejiang Provincial Outstanding Youth Foundation (No. LR13H020001). Conflict of interest: none.

\section{REFERENCES}

1. Kaplanski G. Interleukin-18: biological properties and role in disease pathogenesis. Immunol Rev 2018; 281(1): 138-53.

2. Gu H, Xie M, Xu L, Zheng X, Yang Y, Lv X. The protective role of interleukin-18 binding protein in a murine model of cardiac ischemia/reperfusion injury. Transpl Int 2015; 28(12): 1436-44.

3. Nakanishi K, Yoshimoto $\mathrm{T}$, Tsutsui H, Okamura H. Interleukin18 regulates both Th1 and Th2 responses. Annu Rev Immunol 2001; 19: 423-74. 
4. Xing J, Zhang X, Fan J, Shen B, Men T, Wang J. Association between interleukin-18 promoter variants and tacrolimus pharmacokinetics in Chinese renal transplant patients. Eur J Clin Pharmacol 2015; 71(2): 191-8.

5. Pawlus J, Sierocka A, Tejchman K, et al. The impact of interleukin $12 \mathrm{~B}(1188 \mathrm{~A}>\mathrm{C})$, interleukin $16(-295 \mathrm{~T}>\mathrm{C})$, and interleukin $18(607 \mathrm{C}>\mathrm{A}, 137 \mathrm{G}>\mathrm{C})$ gene polymorphisms on long-term renal transplant function and recipient outcomes. Transplant Proc 2014; 46(6): 2079-82.

6. Li Y, Zou Y, Cai B, et al. The associations of IL-18 serum levels and promoter polymorphism with tacrolimus pharmacokinetics and hepatic allograft dysfunction in Chinese liver transplantation recipients. Gene 2012; 491(2): 251-5.

7. Ono S, Obara H, Takayanagi A, et al. Suppressive effects of interleukin-18 on liver function in rat liver allografts. J Surg Res 2012; 176(1): 293-300.

8. Ozsoy M, Gonul Y, Bal A, et al. Effect of IL-18 binding protein on hepatic ischemia-reperfusion injury induced by infrarenal aortic occlusion. Ann Surg Treat Res 2015; 88(2): 92-9.

9. Yoshimoto T, Takeda K, Tanaka T, et al. IL-12 up-regulates IL-18 receptor expression on T cells, Th1 cells, and B cells: synergism with IL-18 for IFN-gamma production. J Immunol 1998; 161(7): 3400-7.

10. Reddy P. Interleukin-18: recent advances. Curr Opin Hematol 2004; 11(6): 405-10.

11. Li P, Li YL, Li ZY, et al. Cross talk between vascular smooth muscle cells and monocytes through interleukin-1 $\beta /$ interleukin-18 signaling promotes vein graft thickening. Arterioscler Thromb Vasc Biol 2014; 34(9): 2001-11.

12. Dinarello CA. Interleukin-18 and the treatment of rheumatoid arthritis. Rheum Dis Clin North Am 2004; 30(2): 417-434 (ix).

13. Hejr S, Karimi MH, Sabet S, et al. Polymorphism of the IL-18 and CD40 genes and liver transplant outcome in Iranian patients. Int $J$ Organ Transplant Med 2014; 5(4): 149-54.

14. Striz I. Cytokines of the IL-1 family: recognized targets in chronic inflammation underrated in organ transplantations. Clin Sci 2017 ; 131(17): 2241-56

15. Tsutsui H, Nakanishi K. Immunotherapeutic applications of IL-18. Immunotherapy 2012; 4(12): 1883-94.
16. Yamagami H, Kitagawa K, Hoshi T, et al. Associations of serum IL18 levels with carotid intima-media thickness. Arterioscler Thromb Vasc Biol 2005; 25(7): 1458-62.

17. Sirota JC, Walcher A, Faubel S, et al. Urine IL-18, NGAL, IL-8 and serum IL-8 are biomarkers of acute kidney injury following liver transplantation. BMC Nephrol 2013; 14: 17.

18. Gonul Y, Kazandi S, Kocak A, et al. Interleukin-18 binding protein pretreatment attenuates kidney injury induced by hepatic ischemia reperfusion. Am J Med Sci 2016; 352(2): 200-7.

19. Sung WC, Yu HP, Tsai YF, Chung PC, Lin CC, Lee WC. The ratio of plasma interleukin-18 is a sensitive biomarker for acute kidney injury after liver transplantation. Transplant Proc 2014; 46(3): 816-7.

20. Pulitano C, Ho P, Verran D, et al. Molecular profiling of postreperfusion milieu determines acute kidney injury after liver transplantation: a prospective study. Liver Transpl 2018. Doi: 10.1002/lt.25178 [Epub ahead of print].

21. Wyburn K, Wu H, Chen G, Yin J, Eris J, Chadban S. Interleukin18 affects local cytokine expression but does not impact on the development of kidney allograft rejection. Am J Transplant 2006; 6(11): 2612-21.

22. Wyburn K, Wu H, Yin J, Jose M, Eris J, Chadban S. Macrophagederived interleukin-18 in experimental renal allograft rejection. Nephrol Dial Transplant 2005; 20(4): 699-706.

23. Striz I, Krasna E, Honsova E, et al. Interleukin 18 (IL-18) upregulation in acute rejection of kidney allograft. Immunol Lett 2005; 99(1): 30-5.

24. Parikh CR, Jani A, Melnikov VY, Faubel S, Edelstein CL. Urinary interleukin-18 is a marker of human acute tubular necrosis. Am J Kidney Dis 2004; 43(3): 405-14.

25. Hall IE, Yarlagadda SG, Coca SG, et al. IL-18 and urinary NGAL predict dialysis and graft recovery after kidney transplantation. $J \mathrm{Am}$ Soc Nephrol 2010; 21(1): 189-97.

26. do Nascimento WG, Ciliao DA, Genre J, et al. Genetic polymorphisms of interleukin-18 are not associated with allograft function in kidney transplant recipients. Genet Mol Biol 2014; 37(2): 343-9.

27. Chen J, Li L, An Y, et al. Multiple genetic variants associated with posttransplantation diabetes mellitus in Chinese Han populations. J Clin Lab Anal 2018;32(3). doi: 10.1002/jcla.22308 [Epub 2017 Aug 23]. 